\title{
A field model interpretation of crack initiation and growth behavior in ferroelectric ceramics: change of poling direction and boundary condition
}

\author{
G.C. Sih $^{\mathrm{a}, \mathrm{b}, *}$ \\ ${ }^{a}$ Department of Mechanical Engineering and Mechanics, Lehigh University, Bethlehem, PA 18015, USA \\ b Institute of Mechanics, Chinese Academy of Sciences, Beijing 100080, China
}

\begin{abstract}
Strain energy density expressions are obtained from a field model that can qualitatively exhibit how the electrical and mechanical disturbances would affect the crack growth behavior in ferroelectric ceramics. Simplification is achieved by considering only three material constants to account for elastic, piezoelectric and dielectric effects. Cross interaction of electric field (or displacement) with mechanical stress (or strain) is identified with the piezoelectric effect; it occurs only when the pole is aligned normal to the crack. Switching of the pole axis by $90^{\circ}$ and $180^{\circ}$ is examined for possible connection with domain switching. Opposing crack growth behavior can be obtained when the specification of mechanical stress $\sigma^{\infty}$ and electric field $E^{\infty}$ or $\left(\sigma^{\infty}, E^{\infty}\right)$ is replaced by strain $\epsilon^{\infty}$ and electric displacement $D^{\infty}$ or $\left(\epsilon^{\infty}, D^{\infty}\right)$. Mixed conditions $\left(\sigma^{\infty}, D^{\infty}\right)$ and $\left(\epsilon^{\infty}, E^{\infty}\right)$ are also considered. In general, crack growth is found to be larger when compared to that without the application of electric disturbances. This includes both the electric field and displacement. For the eight possible boundary conditions, crack growth retardation is identified only with $\left(E_{y}^{\infty}, \sigma_{y}^{\infty}\right)$ for negative $E_{y}^{\infty}$ and $\left(D_{y}^{\infty}, \epsilon_{y}^{\infty}\right)$ for positive $D_{y}^{\infty}$ while the mechanical conditions $\sigma_{y}^{\infty}$ or $\epsilon_{y}^{\infty}$ are not changed. Suitable combinations of the elastic, piezoelectric and dielectric material constants could also be made to suppress crack growth.
\end{abstract}

(c) 2002 Published by Elsevier Science Ltd.

\section{Introduction}

Ferroelectric materials such as barium titanate retain their polarization even after an electric field has been withdrawn. Dipoles aligned with the field are thus formed in the material microstructure.

\footnotetext{
*Address: School of Advanced Science and Technology, Xi'an Jiaotong University, P.O. Box 1175, 710049 Xi'an Shaanxi, China. Fax: +86-29-2668028.

E-mail address: gcs@xjtu.edu.cn (G.C. Sih).
}

Parallel orientation of dipoles in the opposite direction can also occur by reversing the electric field until a threshold referred to as the coercive strength is reached. This forms a complete hysteresis loop such that the enclosed area would be proportional to the energy dissipated per unit volume in a full field cycle. The dissipated energy would accumulate and increase at the expense of the stored energy required to maintain continuity of the material. Discontinuities in the form of cracks could occur at all scales from domains to devices when sufficient number of full field cycles are applied. 
Since miniaturized devices are the rule in modern electronic industry rather than the exception, microscopic defects or geometric irregularities could often act as the primary crack initiators. They can no longer be overlooked and need to be regulated in the manufacturing process.

A feasible way to control microdefects requires a knowledge of the conditions under which certain defects are harmful while others are not or may even be beneficial. This depends solely on the operational environment that changes for each application. Reliance on simple test results alone could be inadequate. Effective analytical solutions are still most expedient for determining the critical parameters that control failure. Too much emphases cannot be placed on the judicious combination of field solution with the appropriate failure criterion that decides on the outcome. Such a requirement will be demonstrated in this work for the crack initiation behavior of the class of PZT piezoceramics.

The classical energy release rate criterion and those based on letting the crack extension vanish in the limit assume that all energy are converted to the creation of new fracture surface instantaneously at the same size and time scale. In order to describe fracture damage at different scale levels in a single formulation, it is necessary to consider the multiscale behavior characterized by defect initiation, growth with increasing or decreasing speed followed by unstable propagation or arrest. Each stage could vary to reflect how the same material microstructure might react differently when the macroscopic loading and/or boundary conditions are changed. These considerations emphasize the need to know material behavior under test conditions that may not be the same as that under service. At least two tests are necessary. Fracture behavior study of piezoelectric materials [1-3] should involve the specification of stress accompanied by electric field and strain accompanied by electric displacement or the equivalent that can be easily simulated in tests. Not to mention in addition are the effects of pole alignment relative to defect orientation. Opposing behavior of crack growth can result when the direction of electric field is reversed with reference to poling. In practice, a mix of the two foregoing boundary conditions may prevail.
By tradition, far more emphases have been centered on refining the field solution while failure criterion has been selected mostly by a hit-and-run process. Preference has depended on how well the analytical results agree with test data. The challenge is to test the same criterion for different field solutions under different conditions. One of the objectives of this work is to demonstrate that the phenomenon of crack growth enhancement and retardation can be explained by combining a field solution with the appropriate fracture criterion while the oversimplified criterion may lead to disagreement with test data $[4,5]$ and results that contradict physics $[1,6]$ such as negative energy release rate.

The energy density function plays a fundamental role in the development of theories, not only in mechanics but also in physics. It is positive definite and has less likely of a chance to encounter controversy. The rapid decay character of energy density $\mathrm{d} W / \mathrm{d} V$ or energy per unit volume next to a defect can best describe the multiscale feature of material damage. Fig. 1(a)-(c) inclusive illustrate this feature for the evolution of cracking at the micro-, meso- and macroscale, respectively. Near a crack-like defect, mechanics predicts that $\mathrm{d} W / \mathrm{d} V$ rises rapidly without bound according to $1 / r$ where $r$ is the distance from a sharp line defect. Suppose that the linear dimensions less than 0.1, $0.1-10 \mu \mathrm{m}$ and larger than $10 \mu \mathrm{m}$ are assigned to distinguish the respective length scales of micro, meso and macro. Let $r$ stand for a segment of crack extension. The definition of scale length would then require

$r_{\text {micro }}<r_{\text {meso }}<r_{\text {macro }}$

It followed that

$\left(\frac{\mathrm{d} W}{\mathrm{~d} V}\right)_{\mathrm{c}}^{\text {micro }}>\left(\frac{\mathrm{d} W}{\mathrm{~d} V}\right)_{\mathrm{c}}^{\text {meso }}>\left(\frac{\mathrm{d} W}{\mathrm{~d} V}\right)_{\mathrm{c}}^{\text {macro }}$

where $(\mathrm{d} W / \mathrm{d} V)_{\mathrm{c}}$ corresponds to the critical energy density at fracture under unaxial loading. Note that $\mathrm{d} W / \mathrm{d} V$ represents the area under the true stress and true strain curve. If $(\mathrm{d} W / \mathrm{d} V)^{\text {macro }}$ is in MPa then $(\mathrm{d} W / \mathrm{d} V)^{\text {micro }}$ becomes GPa, a three order of magnitude increase. Although there are no available strength data at the micro- and mesoscale to compare with those at the macroscale, the 


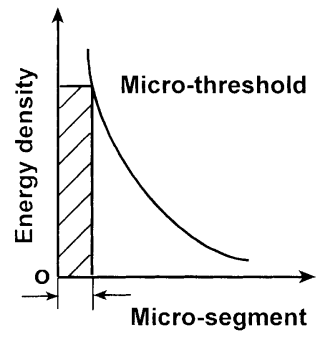

(a) Microscale

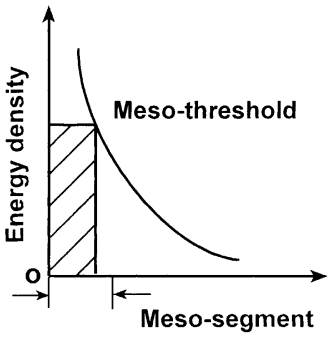

(b) Mesoscale

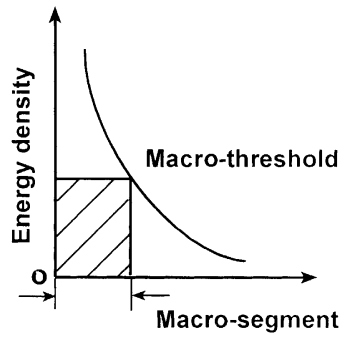

(c) Macroscale

Fig. 1. Energy density elevation near defect at micro-, meso- and macroscale.

curves in Fig. 1(a)-(c) implies that micocracking is likely to occur prior to meso- and macrocracking. These implications are inherently embedded in the decay behavior of $\mathrm{d} W / \mathrm{d} V$. The shaded areas under the curves in Fig. 1 are obtainable from the relation $S=r(\mathrm{~d} W / \mathrm{d} V)$. They could be interpreted as the energy released by crack extension at the different scale levels:

$S^{\text {micro }}<S^{\text {meso }}<S^{\text {macro }}$

The form $\mathrm{d} W / \mathrm{d} V=S / r$ applies to all length scale. This is one of the reasons why the energy density criterion [7-9] could explain the crack growth behavior of piezoceramics caused by microstructure change due to different orientation of poling whereas the energy release rate criterion could not. To be emphasized is that $S / r$ is not necessarily restricted to the asymptotic representation of $\mathrm{d} W /$ $\mathrm{d} V$ unless the additional limit condition $r \rightarrow 0$ is imposed. The equivalence of $r(\mathrm{~d} W / \mathrm{d} V)$ and $S$ arises directly from the fact that the latter is simply the area under the $\mathrm{d} W / \mathrm{d} V$ versus $r$ curve. The $\mathrm{d} W / \mathrm{d} V$ curve in Fig. 1(b) could coincide with that in Fig. 1(a) if the microdamage is not sufficient to alter the stored energy at the mesoscale. The same applies to the curves in Fig. 1(b) and (c). Difference in the $\mathrm{d} W / \mathrm{d} V$ curves at different scales would signify energy dissipated to cause microstructure change.

\section{Electroelastic field model}

Piezoelastic field equations can be greatly simplified for two-dimensional problems where the $x$-component displacement can be assumed to van- ish such that only the $y$-component displacement is used. Such a model has been proposed in [5] for solving crack problems. That is

$u_{x}=0, \quad u_{y}=v(x, y)$

would yield only two nontrivial strain components

$\epsilon_{x}=0, \quad \epsilon_{y}=\frac{\partial v}{\partial y}, \quad 2 \epsilon_{x y}=\frac{\partial v}{\partial x}$

The electric potential $\phi(x, y)$ gives the electric field components:

$E_{x}=-\frac{\partial \phi}{\partial x}, \quad E_{y}=-\frac{\partial \phi}{\partial y}$

Simplification of the transversely isotropic constitutive relations can be made by considering only three constants to describe the elastic, piezoelectric and dielectric properties. The stress and electric displacement components $\sigma_{i j}$ and $D_{i}$ can then be obtained, the forms of which would depend on whether the pole is directed normal or parallel to the crack as illustrated in Fig. 2(a) and (b), respectively. Note that poling is in the three direction. It coincides with the $y$-axis in Fig. 2(a) and $x$-axis in Fig. 2(b).

\subsection{Poling normal to crack}

Referring to Fig. 2(a), the constitutive relations can be written in matrix form as

$$
\left[\begin{array}{c}
\sigma_{x} \\
\sigma_{y} \\
\sigma_{x y} \\
D_{x} \\
D_{y}
\end{array}\right]=\left[\begin{array}{ccccc}
m & 0 & 0 & 0 & e \\
0 & m & 0 & 0 & -e \\
0 & 0 & m & -e & 0 \\
e & 0 & e & \varepsilon & 0 \\
-e & e & 0 & 0 & \varepsilon
\end{array}\right]\left[\begin{array}{c}
\epsilon_{x} \\
\epsilon_{y} \\
2 \epsilon_{x y} \\
E_{x} \\
E_{y}
\end{array}\right]
$$




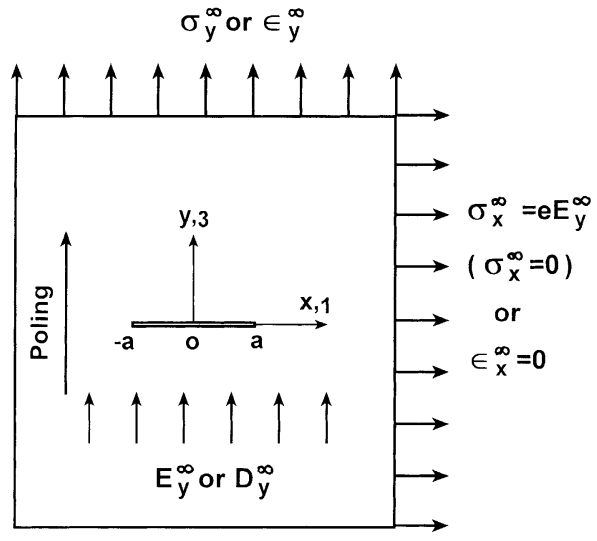

(a) Poling normal to crack

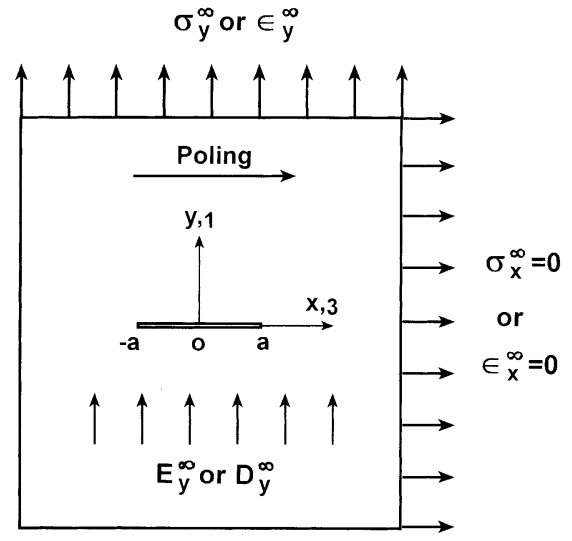

(b) Poling parallel to crack

Fig. 2. Boundary conditions and poling with reference to crack.

in which $m, e$, and $\varepsilon$ are, respectively, the elastic, piezoelectric and dielectric constant. It follows from Eq. (7) and the use of Eqs. (5) and (6) that

$\sigma_{x}=-e \frac{\partial \phi}{\partial y}, \quad \sigma_{y}=\frac{\partial}{\partial y}(m v+e \phi)$,

$\sigma_{x y}=\frac{\partial}{\partial x}(m v+e \phi)$

and

$D_{x}=\frac{\partial}{\partial x}(e v-\varepsilon \phi), \quad D_{y}=\frac{\partial}{\partial y}(e v-\varepsilon \phi)$

To satisfy the equilibrium equations:

$\frac{\partial \sigma_{x y}}{\partial x}+\frac{\partial \sigma_{y}}{\partial y}=0, \quad \frac{\partial D_{x}}{\partial x}+\frac{\partial D_{y}}{\partial y}=0$

the unknowns $v(x, y)$ and $\phi(x, y)$ must be determinable from the Laplace equations

$\nabla^{2} v(x, y)=0, \quad \nabla^{2} \phi(x, y)=0$

For crack problems, the solution can be best stated in the complex plane $z=x+\mathrm{i} y$ such that the singular character of the central crack is expressed by the branch cut $\left(z^{2}-a^{2}\right)^{1 / 2}$. A solution of Eq. (11) can thus be written as

$v=\operatorname{Im}[\Omega(z)], \quad \phi=\operatorname{Im}[\Phi(z)]$

For a crack of length $2 a$ centered along the $x$-axis, $\Omega(z)$ and $\Phi(z)$ are given by

$\Omega(z)=A \sqrt{z^{2}-a^{2}}, \quad \Phi(z)=B \sqrt{z^{2}-a^{2}}$ with $A$ and $B$ being complex constants unless $\sigma_{x y}$ and $D_{x}$ vanish at infinity. Then $A$ and $B$ are real. The constant and linear terms in Eq. (13) can be ignored since they will not affect the distribution of the crack front stress and electric field.

In view of Eqs. (6), (8), (9) and (12), a complex representation of the stress, electric displacement and field components can be made:

$$
\begin{aligned}
& \sigma_{y}+\mathrm{i} \sigma_{x y}=m \Omega^{\prime}(z)+e \Phi^{\prime}(z) \\
& D_{y}+\mathrm{i} D_{x}=e \Omega^{\prime}(z)-\varepsilon \Phi^{\prime}(z) \\
& E_{y}+\mathrm{i} E_{x}=-\Phi^{\prime}(z)
\end{aligned}
$$

Once $\Omega(z)$ and $\Phi(z)$ are known, the crack problem is solved.

Imposed on the crack surface $|x|<a$ are the traction free and impermeable electric boundary conditions:

$\sigma_{y}=\sigma_{x y}=0 \quad$ and $\quad D_{y}=0$ for $|x|<a$

In this simplified model, $\sigma_{x y}=0$ along the crack is not satisfied.

\subsection{Poling parallel to crack}

Suppose that poling is switched $90^{\circ}$ such that it is parallel to the crack as illustrated in Fig. 2(b). The $x$ - and $y$-axis will now coincide with 3 and 1 , respectively. A simplified version of the constitutive equations in matrix form is given by 


$$
\left[\begin{array}{c}
\sigma_{y} \\
\sigma_{x} \\
\sigma_{x y} \\
D_{y} \\
D_{x}
\end{array}\right]=\left[\begin{array}{ccccc}
m & 0 & 0 & 0 & e \\
0 & m & 0 & 0 & -e \\
0 & 0 & m & -e & 0 \\
e & 0 & e & \varepsilon & 0 \\
-e & e & 0 & 0 & \varepsilon
\end{array}\right]\left[\begin{array}{c}
\epsilon_{y} \\
\epsilon_{x} \\
2 \epsilon_{x y} \\
E_{y} \\
E_{x}
\end{array}\right]
$$

Making use of Eqs. (5) and (6), the stresses can be found from Eq. (16) as

$$
\begin{aligned}
& \sigma_{x}=-e \frac{\partial v}{\partial y}, \quad \sigma_{y}=m \frac{\partial v}{\partial y}-e \frac{\partial \phi}{\partial x}, \\
& \sigma_{x y}=m \frac{\partial v}{\partial x}+e \frac{\partial \phi}{\partial y}
\end{aligned}
$$

In the same way, the electric displacement components are also obtained:

$D_{x}=e \frac{\partial v}{\partial y}-\varepsilon \frac{\partial \phi}{\partial x}, \quad D_{y}=e \frac{\partial v}{\partial x}-\varepsilon \frac{\partial \phi}{\partial y}$

Satisfaction of Eq. (10) requires that $v(x, y)$ and $\phi(x, y)$ to satisfy the Laplace equations in Eq. (11). Hence, two complex functions can be introduced to represent

$v=\operatorname{Im}[\Lambda(z)], \quad \phi=\operatorname{Im}[\Psi(z)]$

For the crack problem in Fig. 2(b), $\Lambda(z)$ and $\Psi(z)$ are of the forms

$$
\begin{aligned}
& \Lambda(z)=C \sqrt{z^{2}-a^{2}}+F z, \\
& \Psi(z)=D \sqrt{z^{2}-a^{2}}+G z
\end{aligned}
$$

in which $C, D, \ldots, G$ are complex constants unless shown otherwise.

A complex representation of the stress, electric displacement and field components in terms of $\Lambda(z)$ and $\Psi(z)$ can be written as

$$
\begin{aligned}
& \sigma_{y}+\mathrm{i} \sigma_{x y}=m \Lambda^{\prime}(z)-e \Psi^{\prime}(z) \\
& D_{x}-\mathrm{i} D_{y}=-\left[e \Lambda^{\prime}(z)+\varepsilon \Psi^{\prime}(z)\right] \\
& E_{x}-\mathrm{i} E_{y}=-\Psi^{\prime}(z)
\end{aligned}
$$

The conditions $\sigma_{y}=D_{y}=0$ at $|x|<a$ in Eq. (15) can be satisfied by letting

$m C_{2}-e D_{2}=0, \quad m F_{1}-e G_{1}=0$

and

$e C_{1}+\varepsilon D_{1}=0, \quad F_{2}+G_{2}=0$

in which $C_{j}, D_{j}, \ldots, G_{j}$ for $j=1$ and 2 correspond, respectively, to the real and imaginary parts of $C, D \ldots, G$ in Eq. (20). Imposing the conditions $\sigma_{x y}=0, \quad E_{x}=0 \quad$ for $|z|=\infty$

it is further required that

$m F_{2}-e G_{2}=0, \quad D_{1}+G_{1}=0$

after making use of the first of Eq. (22). The second of Eq. (23) and first of Eq. (24) yield $F_{2}=G_{2}=0$. Eqs. (22), (23) and (25) can thus be used to express the remaining quantities of $C_{j}$, $D_{j}, \ldots, G_{j}$ in terms of two real constants $M$ and $N$ as follows:

$$
\begin{aligned}
& C=\frac{1}{\varepsilon m+e^{2}}(\varepsilon M+\mathrm{i} e N), \quad F=\frac{M e}{m\left(\varepsilon m+e^{2}\right)} \\
& D=\frac{1}{\varepsilon m+e^{2}}(-e M+\mathrm{i} m N), \quad G=\frac{M e}{\varepsilon m+e^{2}}
\end{aligned}
$$

The determination of $M$ and $N$ can be made from the boundary conditions at infinity.

\subsection{Specification of conditions at infinity}

A variety of boundary conditions can be specified at infinity in piezoelasticity. They include the combinations of $\left(\sigma_{y}^{\infty}, E_{y}^{\infty}\right),\left(\epsilon_{y}^{\infty}, D_{y}^{\infty}\right),\left(\sigma_{y}^{\infty}, D_{y}^{\infty}\right)$ and $\left(\epsilon_{y}^{\infty}, E_{y}^{\infty}\right)$. For each case, the poling direction can be aligned normal or parallel to the crack as indicated in Fig. 2(a) and (b). This would give eight possible cases as summarized in Table 1 . In view of the first of Eqs. (8) in this model, a transverse constraint $\sigma_{y}^{\infty}=e E_{y}^{\infty}$ prevails for Case I since $E_{y}^{\infty}$ is one of the primary boundary conditions to be investigated. No transverse constraint exists for the other cases. Cases I-IV referred to as

Table 1

Classification of boundary conditions

\begin{tabular}{llll}
\hline Case no. & $\begin{array}{l}\text { Poling refer- } \\
\text { ence to crack }\end{array}$ & $\begin{array}{l}\text { Boundary } \\
\text { conditions }\end{array}$ & $\begin{array}{l}\text { Transverse } \\
\text { constraint }\end{array}$ \\
\hline & Natural boundary conditions \\
I & Normal & $\left(\sigma_{y}^{\infty}, E_{y}^{\infty}\right)$ & $\sigma_{x}^{\infty}=e E_{y}^{\infty}$ \\
II & Parallel & $\left(\sigma_{y}^{\infty}, E_{y}^{\infty}\right)$ & $\sigma_{x}^{\infty}=0$ \\
III & Normal & $\left(\epsilon_{y}^{\infty}, D_{y}^{\infty}\right)$ & $\epsilon_{x}^{\infty}=0$ \\
IV & Parallel & $\left(\epsilon_{y}^{\infty}, D_{y}^{\infty}\right)$ & $\epsilon_{x}^{\infty}=0$ \\
& Mixed boundary conditions \\
V & Normal & $\left(\sigma_{y}^{\infty}, D_{y}^{\infty}\right)$ & \\
VI & Parallel & $\left(\sigma_{y}^{\infty}, D_{y}^{\infty}\right)$ & $\sigma_{x}^{\infty}=0$ \\
VII & Normal & $\left(\epsilon_{y}^{\infty}, E_{y}^{\infty}\right)$ & $\sigma_{x}^{\infty}=0$ \\
VIII & Parallel & $\left(\epsilon_{y}^{\infty}, E_{y}^{\infty}\right)$ & $\epsilon_{x}^{\infty}=0$ \\
\hline
\end{tabular}


the natural boundary conditions and V-VIII the mixed boundary conditions. A knowledge of how each one of the eight cases would affect failure by cracking of piezoceramics is useful for design considerations.

\section{Criterion for crack initiation and growth}

The observation that crack growth would be enhanced if the electric field is applied in the poling direction was first made in $[10,11]$. Retardation was also found when the poling direction opposes that of the electric field. An assessment of this phenomenon was made in $[12,13]$ by solving the exact piezoelastic field equations and applying the energy density criterion. When the results of failure analysis [4,5] disagrees with those found by tests, there exists the possibilities that the field solution or the failure criterion is at fault. It is also possible that both the field solution and failure criterion are incorrect. To be shown is that the simplified version of the field solution [5] can explain crack growth enhancement and retardation if the energy release rate criterion is replaced by the energy density criterion that has the capability to treat multiscale crack extension behavior.

\subsection{Necessity of scaling damage size and time}

Material tends to separate along a path where volume change is the largest while yielding tends to favor shape change. These two mechanisms of energy dissipation never occur at the same location and time when they are carefully observed in situ. Postmortem examination of fracture corresponds to the terminal time frame of the broken material which may contain evolutionary traces of permanent deformation occurred at different size and time scales. Zone of microplasticity are often found inside the macroplastic enclaves. For analytical model intended to cover the multiscale and evolutionary character of fracture, greater precision in terminology is needed to describe plasticity reflected by nonlinearity and material damage at microscale. Otherwise, the same concept may be expressed by different descriptors.

\subsection{Hierarchical character of damage}

The increment of energy $\Delta W$ stored in an increment of volume $\Delta V$ expressed as a ratio $\Delta W /$ $\Delta V$ becomes increasingly high as it approaches a geometric defect. This is because the local stress and strain can be many times greater than those averaged globally. More specifically, the stress ratio $\sigma_{\ell} / \sigma_{\mathrm{g}}$ would scale according to $(\rho / a)^{\lambda}$ where $\rho$ and $a$ represent the local and global length parameter. Here, $\sigma_{\ell}$ and $\rho$ could be microscopic quantities while $\sigma_{\mathrm{g}}$ and $a$ could be macroscopic. The exponent $\lambda$ is in general complex with $|\lambda|<1$ depending on the local geometry [14] and material inhomogeneity. A defect is thus said to enhance failure initiation because the nearest element acquires a state of hydrostatic tension where volume change is largest. This has been traditionally referred to as "notch tip embrittlement". Material nonhomogeneity and anisotropy could also aggravate the situation.

The choice to a micro-, meso- or macroelement for analysis is arbitrary. Distinction, however, must be made for those quantities such as stress, strain, and energy density that are scale sensitive. That is microstress cannot be equated to macrostress while the total energy $W$ and force do not depend on scale. A discussion of scaling effects connected with the specific surface energy in fracture mechanics can be found in $[15,16]$.

As mentioned earlier, crack initiation and growth criterion cannot be chosen arbitrarily, particularly for piezoelectric materials where cracking may involve multiscale considerations. The energy density criterion [7-9] can best serve this purpose for it reflects the hierarchical character of material damage. It can seek out the location of damage initiation by focusing attention on a unit volume of micro-, meso- or macroelement such that the thresholds for each scale range follow the conditions stated in Eq. (2). Microcracking would thus precede that at the mesoscale followed by macrocracking. Their locations can be determined from the stationary values of $\Delta W / \Delta V$ where the relative minima and maxima would correspond, respectively, to large and small change in volume $\Delta V$. It follows that large $\Delta V$ renders $(\Delta W / \Delta V)_{\min }$ and small $\Delta V$ renders $(\Delta W / \Delta V)_{\max }$. Among many 
of the minima and maxima, there exists one maximum of $(\Delta W / \Delta V)_{\min }$ and one maximum of $(\Delta W / \Delta V)_{\max }$. Their locations are assumed to coincide with the sites of fracture and yield initiation in the energy density criterion [7-9].

\subsection{Variable crack growth steps}

A crack can be modelled to grow in steps as a combination of increasing segments $r_{1}<r_{2}<\cdots$ or decreasing segments $r_{1}>r_{2}>\cdots$. The size of each segment is dictated by the energy released. This is shown graphically in Fig. 3(a) for $S_{1}<$ $S_{2}<\cdots$ and in Fig. 3(b) for $S_{1}>S_{2}>\cdots$ assuming that growth occurs at a critical energy density $(\mathrm{d} W / \mathrm{d} V)_{\mathrm{c}}$ which can vary for each step according to the resistance change of the local material. Stated mathematically, the variable growth condition can be written for constant $(\mathrm{d} W / \mathrm{d} V)_{\mathrm{c}}$ as

$$
\left(\frac{\mathrm{d} W}{\mathrm{~d} V}\right)_{\mathrm{c}}=\frac{S_{1}}{r_{1}}=\frac{S_{2}}{r_{2}}=\cdots=\frac{S_{j}}{r_{j}}=\cdots=\frac{S_{\mathrm{c}}}{r_{\mathrm{c}}} \text { or } \frac{S_{0}}{r_{0}}
$$

Under constant stress, a crack would eventually reach $S_{\mathrm{c}}$ that triggers the onset of unstable fracture. Constant strain would lead to crack arrest at $S_{0}$. The corresponding ligaments are $r_{\mathrm{c}}$ and $r_{0}$. Eq. (27) can be applied at the microscale, mesoscale or macroscale by observing the conditions defined in Eqs. (1)-(3) inclusive. It will be used to determine how crack steps would alter when the direction of electric field or displacement is changed with reference to that of poling. Particular attention will also be given to the ways with which the applied mechanical stress and strain would interact with the external electric field and displacement.

\subsection{Energy density function}

Application of the foregoing concepts requires a knowledge of the energy density function. For this model, $\mathrm{d} W / \mathrm{d} V$ takes the form

$$
\frac{\mathrm{d} W}{\mathrm{~d} V}=\frac{1}{2}\left(\sigma_{y} \epsilon_{y}+\sigma_{x y} \epsilon_{x y}\right)+\frac{1}{2}\left(D_{x} E_{x}+D_{y} E_{y}\right)
$$

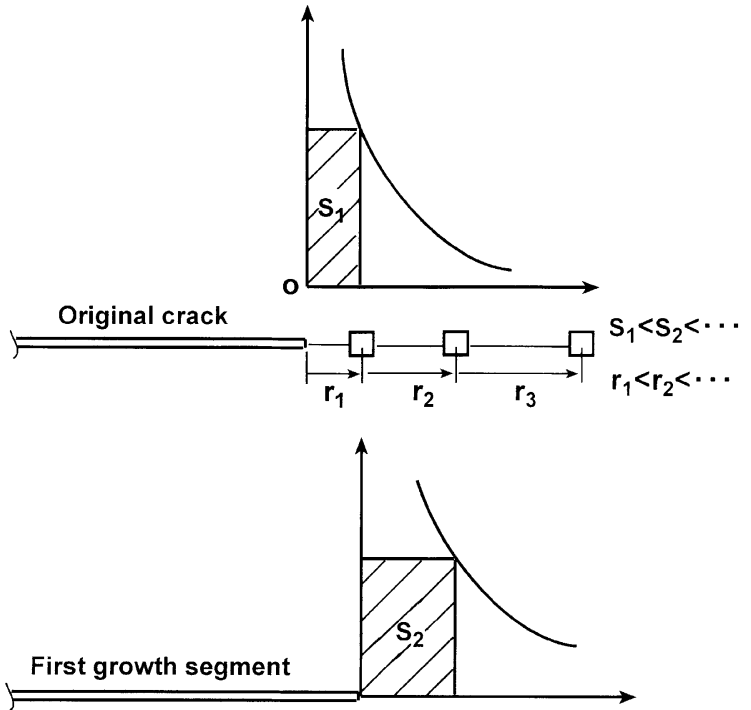

(a) Increasing segment size

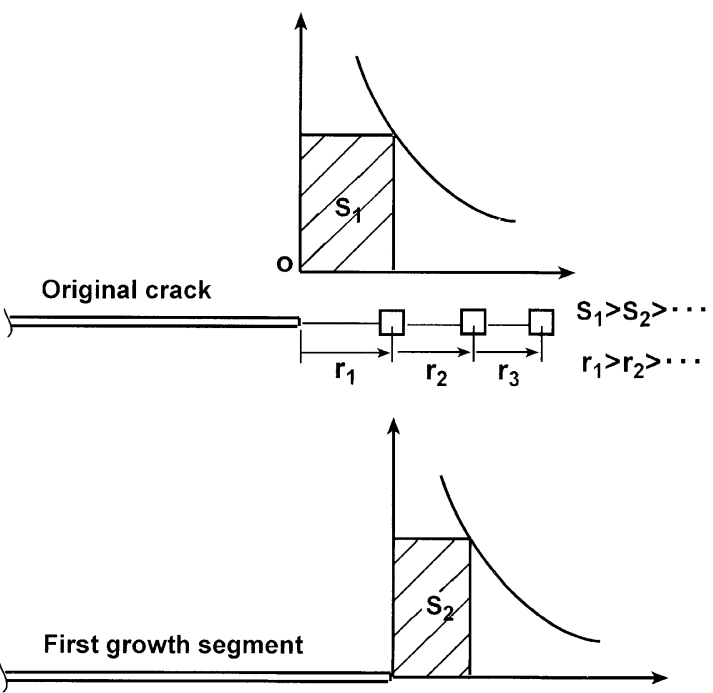

(b) Decreasing segment size

Fig. 3. Variable crack growth segment: (a) increasing segment size, (b) decreasing segment size.

It is not difficult to express $\mathrm{d} W / \mathrm{d} V$ in terms of $A$ and $B$ in Eq. (13) and $M$ and $N$ in Eq. (26) for problems where poling is normal and parallel to the crack, respectively. Without going into the details of algebra, the following expressions are found: 
$S=r \frac{\mathrm{d} W}{\mathrm{~d} V}=\frac{a}{4}\left(m A^{2}+\varepsilon B^{2}\right)$

for poling normal to the crack and

$S=r \frac{\mathrm{d} W}{\mathrm{~d} V}=\frac{1}{4} \frac{1}{\varepsilon m+e^{2}}\left(\varepsilon M^{2}+m N^{2}\right)$

for poling parallel to the crack. In Eqs. (29) and (30), $a$ denotes the half crack length and $r$ the radial distance measured from the crack tip. Here, $S$ is understood to be minimum because the crack initiation path is known to take place in a selfsimilar manner.

\section{Dependency of crack growth on boundary condi- tions and poling}

A crack has been known to extend longer when a positive electric field $E_{y(+)}^{\infty}$ is applied while $\sigma_{y}^{\infty}$ is maintained. A shorter crack extension results for a negative electric field $E_{y(-)}^{\infty}$. This phenomenon $[10,11]$ has been referred to as crack growth enhancement and retardation as illustrated in Fig. 4(a). The reverse phenomenon was also found to occur if strain $\epsilon_{y}^{\infty}$ and electric displacement $D_{y}^{\infty}$ are applied. That is a positive electric displacement $D_{y(+)}^{\infty}$ would lead to a shorter crack extension and $D_{y(-)}^{\infty}$ to a longer crack extension, Fig. 4(b). The assessment involves the application of Eq. (27):

$\frac{S_{j}^{-}}{r_{j}^{-}}=\frac{S_{j}^{0}}{r_{j}^{0}}=\frac{S_{j}^{+}}{r_{j}^{+}}, \quad j=1,2$

where the superscripts,- 0 and + refer, respectively, to the negative, zero and positive electric field or displacement state. Using the zero state as a base reference, Eq. (31) may be written for the conditions in Fig. 4(a) as

$S_{j}^{-}>S_{j}^{0} ; r_{j}^{-}>r_{j}^{0} \quad$ and $S_{j}^{+}<S_{j}^{0} ; r_{j}^{+}<r_{j}^{0}$

It is, therefore, necessary to find the $S$ expressions for those cases in Table 1.

\subsection{Crack normal to poling}

Consider Cases I, III, V and VII in Table 1 where the crack is normal to the pole direction. The constants $A$ and $B$ for these situations are

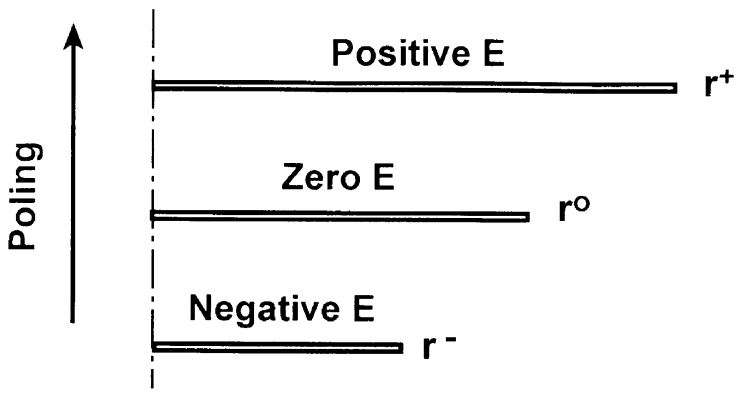

(a) Stress applied

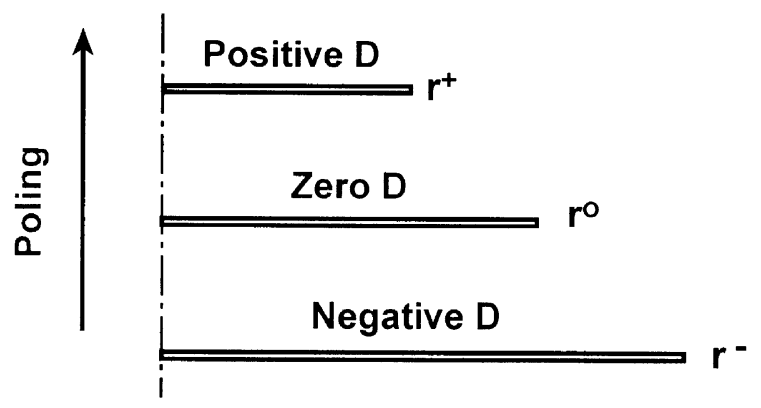

(b) Strain applied

Fig. 4. Crack growth enhancement and retardation with electromechanical coupling: (a) stress applied, (b) strain applied.

evaluated and outlined in Table 2. Defined are the parameters

$$
\begin{aligned}
& p_{\sigma}=E_{y}^{\infty} / \sigma_{y}^{\infty}, \quad q_{\epsilon}=D_{y}^{\infty} / \epsilon_{y}^{\infty}, \\
& p_{\epsilon}=E_{y}^{\infty} / \epsilon_{y}^{\infty}, \quad q_{\sigma}=D_{y}^{\infty} / \sigma_{y}^{\infty}
\end{aligned}
$$

Substituting the expressions $A$ and $B$ of Table 2 into Eq. (29), there results four expressions of $S$ :

- Case I:

$$
S=\frac{a\left(\sigma_{y}^{\infty}\right)^{2}}{4 m}\left[1+2 e p_{\sigma}+\left(e^{2}+\varepsilon m\right) p_{\sigma}^{2}\right]
$$

Table 2

Constants in $S$ factor for crack normal to poling

\begin{tabular}{lll}
\hline Case no. & Constants & \\
\cline { 2 - 3 } & $A$ & $B$ \\
\hline I & $\left(\sigma_{y}^{\infty}+e E_{y}^{\infty}\right) / m$ & $-E_{y}^{\infty}$ \\
III & $\epsilon_{y}^{\infty}$ & $\left(e \epsilon_{y}^{\infty}-D_{y}^{\infty}\right) \varepsilon$ \\
V & $\left(\varepsilon \sigma_{y}^{\infty}+e D_{y}^{\infty}\right) /$ & $\left(e \sigma_{y}^{\infty}-m D_{y}^{\infty}\right) /$ \\
& $\left(e^{2}+\varepsilon m\right)$ & $\left(e^{2}+\varepsilon m\right)$ \\
VII & $\epsilon_{y}^{\infty}$ & $-E_{y}^{\infty}$ \\
\hline
\end{tabular}


- Case III:

$$
S=\frac{a\left(\epsilon_{y}^{\infty}\right)^{2}}{4 \varepsilon}\left[\left(e^{2}+\varepsilon m\right)-2 e q_{\epsilon}+q_{\epsilon}^{2}\right]
$$

- Case V:

$$
S=\frac{a\left(\sigma_{y}^{\infty}\right)^{2}}{4\left(e^{2}+\varepsilon m\right)}\left[\varepsilon+m q_{\sigma}^{2}\right]
$$

- Case VII:

$$
S=\frac{a\left(\epsilon_{y}^{\infty}\right)^{2}}{4}\left[m+\varepsilon p_{\epsilon}^{2}\right]
$$

Note that interaction of $\sigma_{y}^{\infty}$ and $E_{y}^{\infty}$ occurs in Eq. (34) and of $\epsilon_{y}^{\infty}$ and $D_{y}^{\infty}$ occurs in Eq. (35). The sign of $p_{\sigma}$ in Eq. (34) is opposite to that of $q_{\epsilon}$ in Eq. (35). Crack growth enhancement and retardation would thus be reversed when the condition of $\left(\sigma_{y}^{\infty}, E_{y}^{\infty}\right)$ is replaced by $\left(\epsilon_{y}^{\infty}, D_{y}^{\infty}\right)$. This holds only when poling is normal to the crack. Eqs. (36) and (37) show that crack growth enhancement and retardation behavior do not occur for Cases $\mathrm{V}$ and VII where the boundary conditions are mixed.

\subsection{Crack parallel to poling}

Cases II, IV, VI and VIII in Table 3 refer to the situations when the crack is parallel to the pole. Calculations of the energy density factor $S$ involve finding $M$ and $N$ in Eq. (30). The results can be found in Table 3. Similarly, $M$ and $N$ in Table 3 can be inserted into Eq. (30) to yield

\section{- Case II:}

$$
S=\frac{a\left(\sigma_{y}^{\infty}\right)^{2}}{4}\left[\frac{\varepsilon}{e^{2}+\varepsilon m}+\frac{e^{2}+\varepsilon m}{m} p_{\sigma}^{2}\right]
$$

Table 3

Constants in $S$ factor for crack parallel to poling

\begin{tabular}{lll}
\hline Case no. & Constants & \\
\cline { 2 - 3 } & $M$ & $N$ \\
\hline II & $\sigma_{y}^{\infty}$ & $\left(e^{2}+\varepsilon m\right) E_{y}^{\infty} / m$ \\
IV & $m \epsilon_{y}^{\infty}$ & $D_{y}^{\infty}$ \\
VI & $\sigma_{y}^{\infty}$ & $D_{y}^{\infty}$ \\
VIII & $m \epsilon_{y}^{\infty}$ & $\left(e^{2}+\varepsilon m\right) E_{y}^{\infty} / m$ \\
\hline
\end{tabular}

- Case IV:

$$
S=\frac{m a\left(\epsilon_{y}^{\infty}\right)^{2}}{4\left(e^{2}+\varepsilon m\right)}\left[m \varepsilon+q_{\epsilon}^{2}\right]
$$

- Case VI:

$$
S=\frac{a\left(\sigma_{y}^{\infty}\right)^{2}}{4\left(e^{2}+\varepsilon m\right)}\left[\varepsilon+m q_{\sigma}^{2}\right]
$$

- Case VIII:

$$
S=\frac{a\left(\epsilon_{y}^{\infty}\right)^{2}}{4}\left[\frac{\varepsilon m^{2}}{e^{2}+\varepsilon m}+\frac{e^{2}+\varepsilon m}{m} p_{\epsilon}^{2}\right]
$$

Recall that $p_{\sigma} q_{\epsilon}$, etc. are defined in Eq. (33).

\section{Discussion of results}

Numerical calculations will be made for $S$ in Eqs. (34)-(37) and $S$ in Eqs. (38)-(41) for the PZT4 piezoelectric material whose transverse isotropic material properties are given in Table 4 . The average value of $m=6.93 \times 10^{10} \mathrm{~N} / \mathrm{m}^{2}, e=13.64$ $\mathrm{C} / \mathrm{m}^{2}$ and $\varepsilon=5.74 \times 10^{-9} \mathrm{CN} \mathrm{m}^{2}$ will be used for this simplified field model.

\subsection{Behavior of energy density factors}

Plotted in Fig. 5 are the normalized energy density factor $S /\left[\left(\sigma_{y}^{\infty}\right)^{2} a / m\right]$ against the parameter $p_{\sigma}=E_{y}^{\infty} / \sigma_{y}^{\infty}$ for Cases I and II. The curve for Case I decreases to a minimum at $p_{\sigma} \approx 0.025 \mathrm{~V} \mathrm{~m} / \mathrm{N}$; it then rises across $p_{\sigma}=0$ into the positive region of $E_{y}^{\infty} / \sigma_{y}^{\infty}$. This implies that the same state of energy density could exist for two different combination of $E_{y}^{\infty}$ and $\sigma_{y}^{\infty}$ as a ratio. The interactions of $E_{y}^{\infty}$ and $\sigma_{y}^{\infty}$ quantified as a ratio may not be uniquely defined by $S$. The two portions of the curve for Case II are symmetric about $p_{\sigma}=0$ : one for positive $p_{\sigma}$ and one for negative $p_{\sigma}$. Displayed in Fig. 6 are the variations of the normalized quantity $S / m\left(\epsilon_{y}^{\infty}\right)^{2} a$ with $q_{\epsilon}=D_{y}^{\infty} / \epsilon_{y}^{\infty}$. The valley of the curve for Case III is shifted to the positive side of $q_{\epsilon}$. Symmetry about $q_{\epsilon}=0$ is again seen for the curve of Case IV. These characteristics will be reflected by the crack growth behavior based on Eqs. (31) and (32) described in Fig. 4(a) and (b). Refer to Table 5 for the numerical values of the normalized energy density factor $S$ for Cases I-IV. 
Table 4

Material properties of PZT-4 piezoceramic

\begin{tabular}{|c|c|c|c|c|c|c|c|c|}
\hline \multicolumn{4}{|c|}{$\begin{array}{l}\text { Elastic constants } \\
\times 10^{10}\left(\mathrm{~N} / \mathrm{m}^{2}\right)\end{array}$} & \multicolumn{3}{|c|}{ Piezoelectric constants $\left(\mathrm{C} / \mathrm{m}^{2}\right)$} & \multicolumn{2}{|c|}{$\begin{array}{l}\text { Dielectric permittivities } \\
\times 10^{-9}(\mathrm{C} / \mathrm{V} \mathrm{m})\end{array}$} \\
\hline$c_{11}$ & $c_{13}$ & $c_{33}$ & $c_{44}$ & $e_{31}$ & $e_{33}$ & $e_{15}$ & $\varepsilon_{11}$ & $\varepsilon_{33}$ \\
\hline 13.9 & 7.43 & 11.3 & 2.56 & -6.98 & 13.84 & 13.44 & 6.00 & 5.47 \\
\hline
\end{tabular}

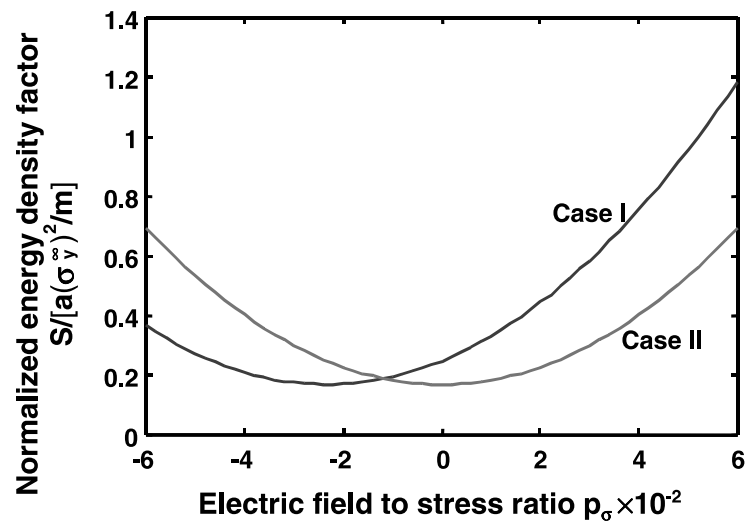

Fig. 5. Normalized energy density factor versus electric field to stress ratio for Cases I and II.

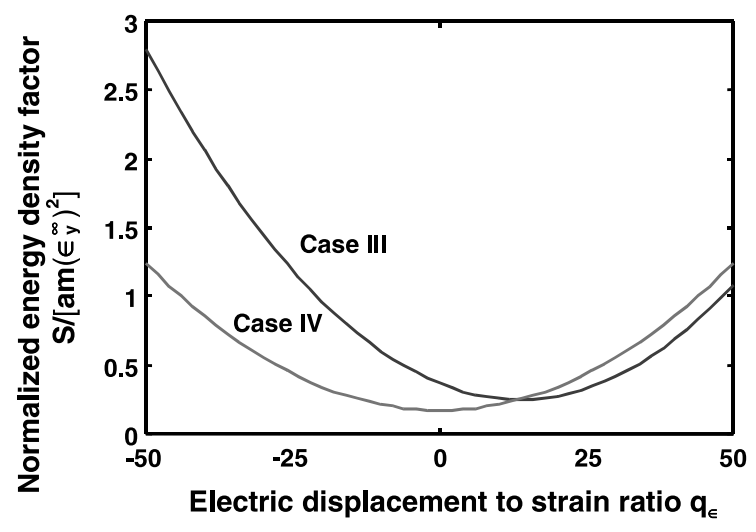

Fig. 6. Normalized energy density factor versus electric displacement to strain ratio for Cases III and IV.

Numerical results of Eqs. (36) and (40) are obtained and shown graphically in Fig. 7. Cases V and VI are identical. This means that the poling direction has no effect on the energy density factor S. Similarly, Eqs. (37) and (41) can be plotted graphically in Fig. 8 for Cases VII and VIII. The curves are similar in trend. Cross product terms of $D_{y}^{\infty}$ and $\sigma_{y}^{\infty}$ or $E_{y}^{\infty}$ and $\epsilon_{y}^{\infty}$ do not appear in Eqs.
Table 5

Normalized energy density factor for Cases I-IV

\begin{tabular}{lllllll}
\hline $\begin{array}{l}p_{\sigma} \\
(\mathrm{V} \mathrm{m} / \mathrm{N})\end{array}$ & \multicolumn{2}{l}{$S /\left[a\left(\sigma_{y}^{\infty}\right)^{2} / m\right]$} & & $q_{\epsilon}$ & \multicolumn{2}{l}{$S /\left[a m\left(\varepsilon_{y}^{\infty}\right)^{2}\right]$} \\
\cline { 2 - 3 } \cline { 5 - 6 } & Case I & Case II & & \multicolumn{2}{l}{$\begin{array}{l}\text { Case } \\
\text { III }\end{array}$} & \multicolumn{1}{l}{ Case } \\
& & & & IV \\
\hline-0.06 & 0.3659 & 0.6954 & -50 & 2.7976 & 1.2414 \\
-0.04 & 0.2106 & 0.4037 & -30 & 1.4480 & 0.5559 \\
-0.03 & 0.1767 & 0.3016 & -10 & 0.6015 & 0.2131 \\
-0.024 & 0.1703 & 0.2543 & 0 & 0.3670 & 0.1703 \\
-0.02 & 0.1719 & 0.2286 & 10 & 0.2583 & 0.2131 \\
0.0 & 0.25 & 0.1703 & 14 & 0.2501 & 0.2543 \\
0.02 & 0.4447 & 0.2286 & 20 & 0.2754 & 0.3417 \\
0.04 & 0.7562 & 0.4037 & 30 & 0.4184 & 0.5559 \\
0.06 & 1.1843 & 0.6954 & 50 & 1.0816 & 1.2414 \\
\hline
\end{tabular}

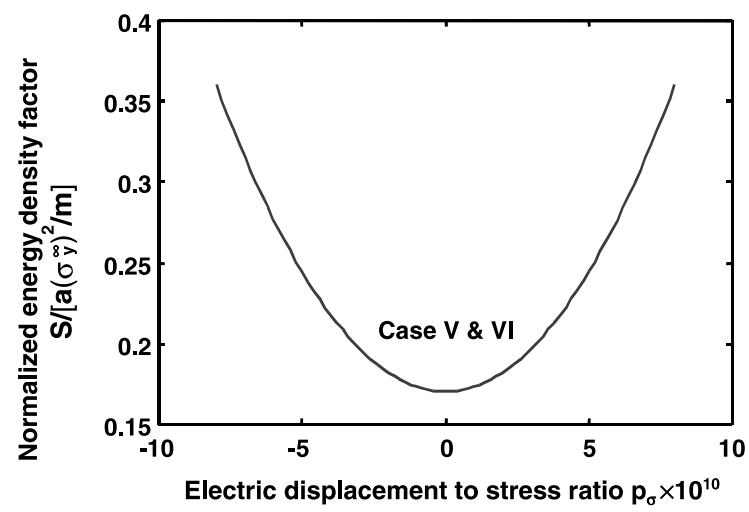

Fig. 7. Normalized energy density factor versus electric displacement to stress ratio for Cases V and VI.

(36), (37), (40) and (41). This behavior holds for Cases V-VIII when poling is parallel to the crack. Numerical values of the curves in Figs. 7 and 8 are given in Table 6.

\subsection{Normalized crack growth segments}

As referred to earlier, $r^{0}$ would stand for the zero field state corresponding to the absence of 


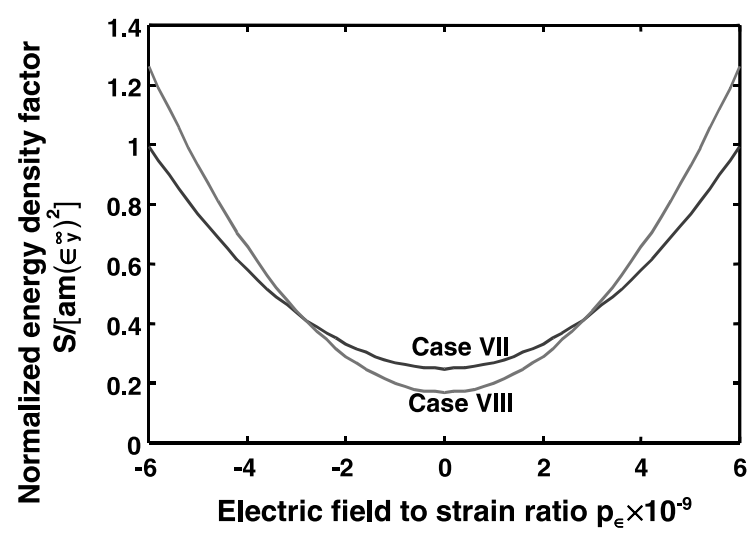

Fig. 8. Normalized energy density factor versus electric field to strain ratio for Cases VII and VIII.

electric field and displacement. It will be different for each case and used as a normalization factor for the crack growth segment as $r / r^{0}$. Crack growth enhancement correspond to $r / r^{0}>1$ and retardation to $r / r^{0}<1$.

Applying Eq. (31) to the data of Fig. 5, the normalized crack growth segments $r / r^{0}$ can be found. They are plotted against $p_{\sigma}=E_{y}^{\infty} / \sigma_{y}^{\infty}$. For Case I in Fig. 9, $r / r^{0}<1$ (retardation) corresponds to negative electric field and $r / r^{0}>1$ (enhancement) to positive electric field. This agrees with the experimental observation in $[10,11]$ and the analytical predictions $[12,13]$ in which the exact field solutions of piezoelasticity was obtained. The results in Fig. 9 show that the approximate field solution of [5] could predict the correct crack growth behavior for Case I if the energy density

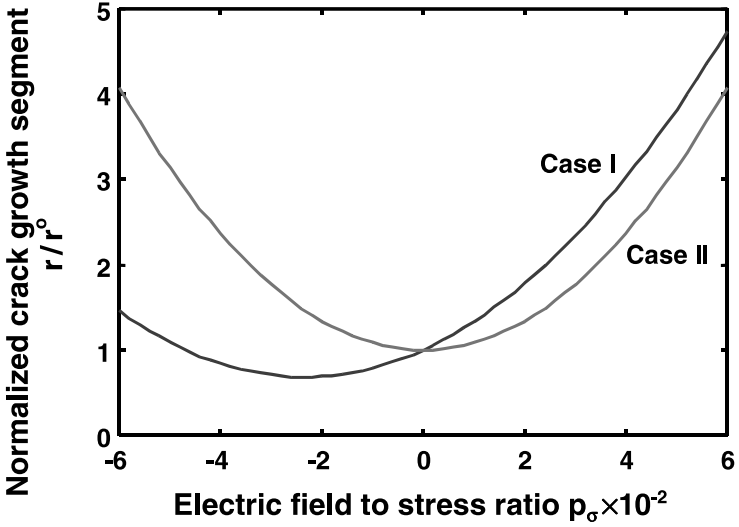

Fig. 9. Normalized crack growth segment versus electric field to stress ratio for Cases I and II.

criterion is used instead of that based on the energy release rate. The objection of a negative energy release rate can thus be removed. This applies also to the work in [6]. Crack growth is always enhanced for Case II regardless of the sign of the applied electric field $E_{y}^{\infty}$. A symmetric $r / r^{0}$ versus $p_{\sigma}$ curve is thus obtained. Fig. 10 shows the results for Cases III and IV. Specification of $D_{y}^{\infty} / \epsilon_{y}^{\infty}$ yields the opposite behavior of crack growth as compared with that of applying $E_{y}^{\infty} / \sigma_{y}^{\infty}$ in Fig. 9. Note that $r / r^{0}>1$ and $r / r^{0}<1$ now refer to negative and positive external disturbance where electric displacement $D_{y}^{\infty}$ is applied. This is exhibited by the curve for Case III. Case IV again yields crack growth enhancement for both positive and negative $q_{\epsilon}$. Summarized in Table 7 are the numerical values of $r / r^{0}$ for Cases I-IV.

Table 6

Normalized energy density factor for Cases V-VIII

\begin{tabular}{|c|c|c|c|c|c|}
\hline \multirow[t]{2}{*}{$q_{\sigma} \times 10^{10}(\mathrm{C} / \mathrm{N})$} & \multicolumn{2}{|c|}{$S /\left[a\left(\sigma_{y}^{\infty}\right)^{2} / m\right]$} & \multirow[t]{2}{*}{$p_{\epsilon} \times 10^{-9}(\mathrm{~V} / \mathrm{m})$} & \multicolumn{2}{|c|}{$S /\left[a m\left(\varepsilon_{y}^{\infty}\right)^{2} / m\right]$} \\
\hline & Case V & Case VI & & Case VII & Case VIII \\
\hline-8.0 & 0.3603 & 0.3603 & -6.0 & 0.9948 & 1.2638 \\
\hline-6.0 & 0.2772 & 0.2772 & -4.0 & 0.5810 & 0.6563 \\
\hline-4.0 & 0.2178 & 0.2178 & -2.0 & 0.3328 & 0.2918 \\
\hline-2.0 & 0.1822 & 0.1822 & 0.0 & 0.25 & 0.1703 \\
\hline 0.0 & 0.1703 & 0.1703 & 2.0 & 0.3328 & 0.2918 \\
\hline 2.0 & 0.1822 & 0.1822 & 4.0 & 0.5810 & 0.6563 \\
\hline 4.0 & 0.2178 & 0.2178 & 6.0 & 0.9948 & 1.2638 \\
\hline 6.0 & 0.2772 & 0.2772 & & & \\
\hline 8.0 & 0.3603 & 0.3603 & & & \\
\hline
\end{tabular}




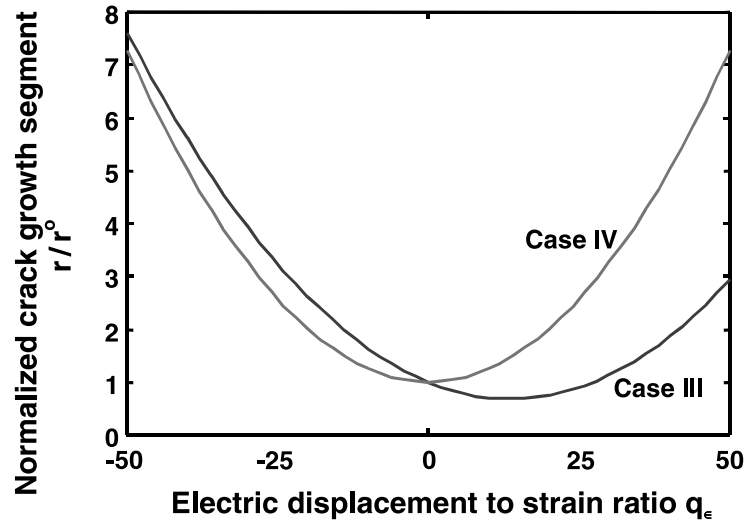

Fig. 10. Normalized crack growth segment versus electric displacement to strain ratio for Cases III and IV.

Table 7

Normalized crack growth segment for Cases I-IV

\begin{tabular}{|c|c|c|c|c|c|}
\hline \multirow{2}{*}{$\begin{array}{l}p_{\sigma} \\
(\mathrm{Vm} / \mathrm{N})\end{array}$} & \multicolumn{2}{|l|}{$r / r^{0}$} & \multirow{2}{*}{$\begin{array}{l}q_{\epsilon} \\
\left(\mathrm{C} / \mathrm{m}^{2}\right)\end{array}$} & \multicolumn{2}{|l|}{$r / r^{0}$} \\
\hline & Case I & Case II & & $\begin{array}{l}\text { Case } \\
\text { III }\end{array}$ & $\begin{array}{l}\text { Case } \\
\text { IV }\end{array}$ \\
\hline-0.06 & 1.4637 & 4.0839 & -50 & 7.6223 & 7.2903 \\
\hline-0.04 & 0.8424 & 2.3706 & -30 & 3.9451 & 3.2645 \\
\hline-0.03 & 0.7067 & 1.7710 & -10 & 1.6389 & 1.2516 \\
\hline-0.024 & 0.6814 & 1.4934 & 0 & 1.0 & 1.0 \\
\hline-0.02 & 0.6878 & 1.3427 & 10 & 0.7038 & 1.2516 \\
\hline 0.0 & 1.0 & 1.0 & 14 & 0.6814 & 1.4932 \\
\hline 0.02 & 1.779 & 1.3427 & 20 & 0.7505 & 2.0065 \\
\hline 0.04 & 3.0248 & 2.3706 & 30 & 1.1398 & 3.2645 \\
\hline 0.06 & 4.7373 & 4.0839 & 50 & 2.9469 & 7.2903 \\
\hline
\end{tabular}

Table 8

Normalized crack growth segment for Cases V-VIII

\begin{tabular}{|c|c|c|c|c|c|}
\hline \multirow[t]{2}{*}{$q_{\sigma} \times 10^{10}(\mathrm{C} / \mathrm{N})$} & \multicolumn{2}{|l|}{$r / r^{0}$} & \multirow[t]{2}{*}{$p_{\epsilon} \times 10^{-9}(\mathrm{~V} / \mathrm{m})$} & \multicolumn{2}{|l|}{$r / r^{0}$} \\
\hline & Case V & Case VI & & Case VII & Case VIII \\
\hline-8.0 & 2.1160 & 2.1160 & -6.0 & 3.9792 & 7.4214 \\
\hline-6.0 & 1.6277 & 1.6277 & -4.0 & 2.3241 & 3.8540 \\
\hline-4.0 & 1.2790 & 1.2790 & -2.0 & 1.3310 & 1.7135 \\
\hline-2.0 & 1.0697 & 1.0697 & 0.0 & 1.0 & 1.0 \\
\hline 0.0 & 1.0 & 1.0 & 2.0 & 1.3310 & 1.7135 \\
\hline 2.0 & 1.0697 & 1.0697 & 4.0 & 2.3241 & 3.8540 \\
\hline 4.0 & 1.2790 & 1.2790 & 6.0 & 3.9792 & 7.4214 \\
\hline 6.0 & 1.6277 & 1.6277 & & & \\
\hline 8.0 & 2.1160 & 2.1160 & & & \\
\hline
\end{tabular}

Results of $r / r^{0}$ for Cases V-VIII are displayed in Figs. 11 and 12. All the curves are normalized

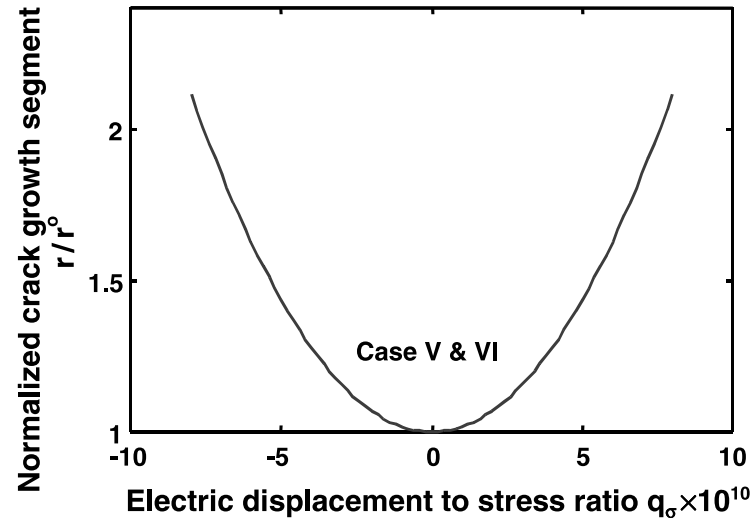

Fig. 11. Normalized crack growth segment versus electric displacement to stress ratio for Cases V and VI.

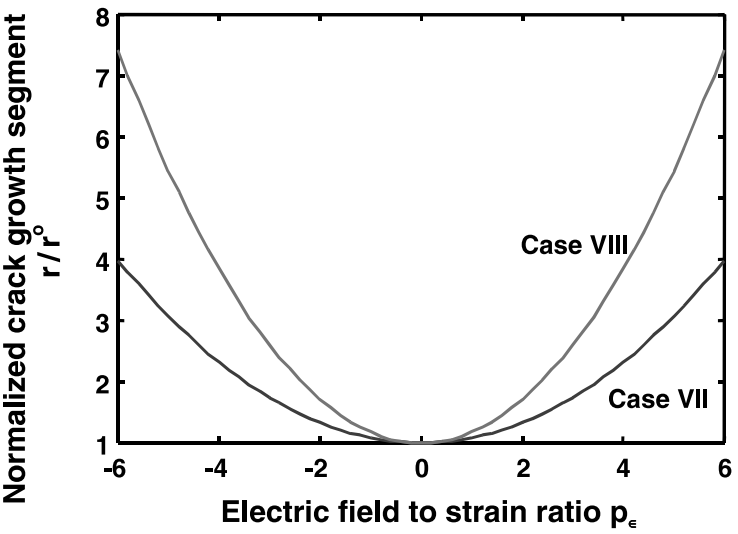

Fig. 12. Normalized crack growth segment versus electric field to strain ratio for Cases VII and VIII. with their vertices at $r / r^{0}=1.0$ and $p_{\epsilon}=0$ or $q_{\epsilon}=0$. The mixed boundary conditions as defined 
in Table 1 are undesirable. They all tend to enhance crack growth for positive and negative electric disturbances. Table 8 gives the numerical results of $r / r^{0}$; they refer to Cases V-VIII.

\section{Concluding remarks}

Effective analytical solutions are obtained from a simplified field model to explain the basic cracking behavior of piezoceramics that would have otherwise required voluminous numerical data for solving the exact but complicated field equations to sort out the underlying physical implications. Electric disturbances are shown to enhance crack growth for majority of the boundary conditions. This is undesirable. Retardation of crack growth are identified with two electromechanical loadings with poling aligned normal to the crack. They involve the application of $\left(\sigma^{\infty}, E^{\infty}\right)$ for negative $E^{\infty}$ and $\left(\epsilon^{\infty}, D^{\infty}\right)$ for positive $D^{\infty}$.

The above interpretations follow from the quadratic form of $S$ in Eq. (34) for Case I and Eq. (35) for Case II. Note from Fig. 5 that two negative states of $\left(\sigma^{\infty}, E^{\infty}\right)$ could prevail for $r / r^{0}<1$ under the same $S$. In other words, electromechanical coupling effects could yield the same energy density state even though the mechanical stress and electric field for the two situations are different. The same can be said for Case III in Fig. 6. Here, two positive states of $\left(\epsilon^{\infty}, D^{\infty}\right)$ are found for $r / r^{0}<1$ with the same $S$. For the other cases, $S$ acquires a canonical form. This means that all the $S$ curves are symmetrical about $p_{\sigma}=p_{\epsilon}=q_{\sigma}=$ $q_{\epsilon}=0$. The same $S$ can correspond to only one positive or one negative state of $p_{\sigma}, p_{\epsilon}, \ldots, q_{\epsilon}$. This is reminiscent of applying the $S$ criterion to crack initiation under uniaxial tension and compression where the same $S$ applies to $\pm \sigma^{\infty}$ since $S \sim\left( \pm \sigma^{\infty}\right)^{2}$. Crack initiation behavior for the two loadings is distinguished from the roots of the characteristic equation of $S$ expressed in terms of the crack initiation angles: one for $\sigma^{\infty}$ and one for $-\sigma^{\infty}$. This can be best illustrated under mixed mode when the crack is inclined to the external mechanical and/or electrical load. This should not differ for piezoelasticity. A discussion of this can be found in [13].
Specific numerical results are made available for PZT-4 only. However, the method of approach applies in general to poled barium-titanate (Ba$\mathrm{TiO}_{3}$ ) and lead-zirconate-titanate. These materials exhibit macroscopic piezoelectricity when they are poled. To reiterate, it is the electromechanical coupling effect that contributes to the enhancement and retardation of crack growth [17]. The cross product terms $\sigma^{\infty} E^{\infty}$ in Eq. (34) and $\epsilon^{\infty} D^{\infty}$ in Eq. (35) are responsible; they are multiplied by the piezoelastic constant $e$. The sign of $E^{\infty}$ and $D^{\infty}$ would dictate whether an increase in $e$ would enhance or retard crack growth. The dimensionless parameter $e / \sqrt{\varepsilon m}$ gives an estimate of the electromechanical coupling strength ranging from 0.1 to 1.0. The lower and upper limit apply, respectively, to the PZT- 6 and PZT-4 or -5 group of piezoceramics. For PZT-4, $m \sim 10^{11} \mathrm{~N} / \mathrm{m}^{2}, e \sim 10$ $\mathrm{C} / \mathrm{m}^{2}$ and $\varepsilon \sim 10^{-9} \mathrm{C} / \mathrm{V} \mathrm{m}^{2}$. This gives $e / \sqrt{\varepsilon m}=1.0$ for PZT-4. The PZT-6 group is not as desirable for suppressing crack growth because $e$ is one third lower while $\varepsilon$ and $m$ are the same as those for PZT4. It follows that $e / \sqrt{\varepsilon m}=0.33$ applies to PZT-6 whose electromechanical coupling capability is less by a factor of 3 .

An in-depth understanding of the hierarchical character of crack tip damage may require a refined field model that could assess cracking at the micro-, meso-, and macroscale. Energy dissipated at the different scale levels needs to be identified with the physical mechanisms. To this end, the distribution of dilatational energy density in relation to that caused by distortion has been used in $[19,20]$. Crack bifurcation was discovered to initiate at distances of the order of $r / a \sim 10^{-6}$. This microbranching phenomenon associated with dilatation differs from that assumed in the classical theory of dislocation where shear is taken to be the cause of branching. As $r / a$ increases to the order of $r / a \sim 10^{-1}$ the zones of dominant dilatation would fold into a single path along the line of Mode I symmetry where the crack grows. This dual-scale feature was predicted in a single formulation from the energy density criterion. Linear piezoelasticity and a sharp crack configuration were used for a homogeneous medium. Finite crack front radius and inhomogeneity material are expected to yield multiscale characteristics. 
In two dimensions, at least two space variable are required to locate the site of failure initiation. Stationary values of the energy density function need to be determined using both $r$ and $\theta$ as the cylindrical polar coordinates. There is no conceptual difficulty to apply continuum mechanics at the different scales because the theory addresses only relative scale effects, say the local and global. The former and latter could represent the micro- and macroscale, respectively. The relation $\left(\sigma_{\ell} / \sigma_{\mathrm{g}}\right)=$ $(\rho / 4 a)^{1 / 2}$ for example applies to a macrocrack of length $2 a$ with $\rho$ being the microsize local radius. The crack tip microstress can thus be scaled to the remote macrostress by using the factor $(\rho / 4 a)^{1 / 2}$. Appropriate adjustment of the material parameters in continuum theories, however, should be made prior to developing scaling relations. Refer to [18-20] for implications associated with using continuum mechanics in contrast to theories that assume atoms as the basic material constituents.

\section{References}

[1] Y.E. Pak, Linear electro-elastic fracture mechanics of piezoelectric material, Int. J. Fract. (54) (1992) 79-100.

[2] G.C. Sih, Z.F. Song, Damage analysis of tetragonal perovskite structure ceramics implicated by asymptotic field solutions and boundary conditions, J. Theor. Appl. Fract. Mech. 38 (2002) 15-36.

[3] G.C. Sih, B. Liu, Z.F. Song, H.F. Ren, Anti-plane shear crack growth in piezoceramics: change of electric field and displacement direction, in: Proceedings of the 10th International Congress on Fracture, Hawaii, December 2-6, 2001, p. 714.

[4] S. Park, C.T. Sun, Fracture criterion of piezoelectric ceramics, J. Am. Ceram. Soc. 78 (1995) 1475-1480.

[5] H.J. Gao, T.Y. Zhang, P. Tong, Local and global energy release rates for an electrically yielded crack in a piezoelectric ceramic, J. Mech. Phys. Solids 45 (1997) 491-510.

[6] Z. Suo, C.M. Kuo, D.M. Barnett, J.R. Willis, Fracture mechanics for piezoelectric ceramics, J. Mech. Phys. Solids 40 (1992) 739-765.
[7] G.C. Sih, Strain energy density factor applied to mixed mode crack problems, Int. J. Fract. Mech. 10 (1974) 305321.

[8] G.C. Sih, Fracture mechanics of engineering structural components, in: G.C. Sih, L. Faria (Eds.), Fracture Mechanics Methodology, vol. 1, Martinus Nijhoff Publishers, The Netherlands, 1984, pp. 35-101.

[9] G.C. Sih, Mechanics of Fracture Initiation and Propagation, Kluwer Academic Publishers, The Netherlands, 1991.

[10] Y.E. Pak, A. Tobin, On the electric field effects in fracture of piezoelectric materials, Mechanics of Electromagnetic Materials and Structures, AMD-vol. 161/MD_vol. 42, ASME, Cleaveland, OH, 1993.

[11] A. Tobin, Y.E. Pak, Effects of electric fields on fracture behavior of PZT ceramics, in: V.K. Varadan (Ed.), Smart Materials, SPIE, vol. 1916, 1993, pp. 77-86.

[12] Z. Zuo, G.C. Sih, Energy density formulation and interpretation of cracking behavior for piezoelectric ceramics, J. Theor. Appl. Fract. Mech. 34 (1) (2000) 17-33.

[13] G.C. Sih, J.Z. Zuo, Multiscale behavior of crack initiation and growth in piezoelectric ceramics, J. Theor. Appl. Fract. Mech. 34 (2000) 123-141.

[14] G.C. Sih, J.W. Ho, Sharp notch fracture strength characterized by critical energy density, J. Theor. Appl. Fract. Mech. 16 (3) (1991) 179-214.

[15] G.C. Sih, Introduction to a series on mechanics of fracture, in: Methods of Analysis and Solutions of Crack Problems, Mechanics of Fracture, vol. I, Noordhoff International Publishing, The Netherlands, 1973, pp. 9-12.

[16] G.C. Sih, Some basic problems in fracture mechanics and new concepts, J. Eng. Fract. Mech. 5 (1973) 365377.

[17] G.C. Sih, Effect of electric field reversal on crack growth behavior of poled piezoelectric ceramic, in: Proceedings of the 10th International Congress on Fracture, Hawaii, December 2-6, 2001, p. 711.

[18] G.C. Sih, Implication of scaling hierarchy associated with nonequilibrium: field and particulate, Prospects of Mesomechanics in the 21st Century (Special issue), J. Theor. Appl. Fract. Mech. 37 (2001) 335-369.

[19] G.C. Sih, B. Liu, Meso-frature mechanics: a necessary link, Prospects of Mesomechanics in the 21st Century, J. Theor. Appl. Fract. Mech. 37 (2002) 371-395.

[20] G.C. Sih, A special theory of crack propagation, in: Method of Analysis and Solutions of Crack Problems, Mechanics of Fracture, vol. I, Noordhoff Publishing, The Netherlands, 1973. 